\title{
Obesidad y supervivencia a los 5 años en pacientes con insuficiencia renal crónica
}

\section{avanzada}

Francisco Barbosa Martín* - María Ángeles Guerrero Riscos** - Jesús Lucas Martín Espejo* Luisa Soler Junco* - Rosa Galeano Macías*** - Rafael Montes Delgado** - Ana Ruiz Fernández**

*Diplomado en Enfermería - ** Médico Nefrólogo - ***Auxiliar Enfermería

Servicio de Nefrología. Unidad de Prediálisis y diálisis domiciliaria. Hospital Virgen del Rocío. Sevilla

\section{Resumen}

En los últimos años se han publicado diversos estudios en pacientes con insuficiencia renal crónica en hemodiálisis en los que se demuestra "paradójicamente" que, al contrario que en la población general, la obesidad, medida a través del índice de masa corporal, se relaciona con mejor supervivencia. En este trabajo hemos estudiado la influencia de la obesidad en la supervivencia a los 5 años en pacientes con insuficiencia renal en Prediálisis.

La muestra estaba formada por 160 pacientes, vistos por primera vez en la consulta de Prediálisis desde el 1 de septiembre de 1998 a 31 de octubre de 1999. Se recogieron de la Historia de Enfermería los datos para calcular el IMC (peso $(\mathrm{Kg}) /$ altura2) en la primera (basal) y última (final) visita en Consulta Prediálisis. Consideramos obeso al paciente con IMC $\geq 30$.

De los 157 pacientes que conocimos la evolución a los 5 años: 85 (54\%) permanecían vivos: 19 seguí-

Correspondencia:

Francisco Barbosa Martín

Sector Triángulo, Bloque $11,5^{\circ} \mathrm{C}$

41089 Montequinto (Sevilla) an en Prediálisis, 43 en diálisis y 23 con un trasplante funcionante; Los pacientes con un IMC $\geq 30$ tenían una supervivencia en meses inferior a los no obesos ( $53 \geq 24$ vs $61 \geq 19$ meses $p=0,04$ ), es decir, la obesidad, definida como IMC $\geq 30$, influye negativamente en la supervivencia a los 5 años en nuestra población de pacientes con insuficiencia renal avanzada atendidos en la Consulta Prediálisis.

Palabras clave

SUPERVIVENCIA

OBESIDAD

ÍNDICE DE MASA CORPORAL

Obesity and survival after 5 years in patients with advanced chronic renal insufficiency

\section{Abstract}

In recent years a number of studies have been published on patients with chronic renal insufficiency undergoing haemodialysis in which it is shown "paradoxically" that, contrary to what occurs in the population in general, obesity, measured through the body mass index, is related to better survival. Here we have studied the influence of obesity on survival after 5 years in patients with renal insufficiency in predialysis. 
The sample was made up of 160 patients, seen for the first time in the predialysis clinic between 1 September 1998 and 31 October 1999. The data required to calculate their BMI (weight $(\mathrm{kg}) /$ height2) was taken from the nursing case history on the first (basal) and last (final) predialysis visit. We considered patients with $B M I \geq 30$ to be obese.

Of the 157 patients whose evolution after 5 years is known to us: 85 (54\%) were still alive: 19 were still in predialysis, 43 on dialysis and 23 with a functioning transplant. The patients with a BMI $\geq 30$ had a lower survival in months than the non-obese patients $(53 \pm 24$ compared to $61 \pm 19$ months $p=$ 0.04 ), in other words, obesity defined as IMC $\geq 30$ has a negative effect on survival after 5 years in our population of patients with advanced renal insufficiency treated in the predialysis clinic.

\section{Key words}

SURVIVAL

OBESITY

BODY MASS INDEX

CHRONIC RENAL INSUFFICIENCY

\section{Introducción}

La labor de Enfermería en una consulta de pacientes con insuficiencia renal crónica (IRC) avanzada (Prediálisis) tiene múltiples facetas, una de las más importantes es la valoración y seguimiento del estado de nutrición (EN). Las medidas antropométricas (MAM) son un método clásico de valoración nutricional. Uno de sus inconvenientes es su falta de reproductilidad cuando son medidas por distintos observadores $^{1}$. Por otro lado, el índice de masa corporal (IMC), que se obtiene de las dos medidas antropométricas de reproductilidad y medida más fácil y asequibles (al dividir el peso por el cuadrado de la altura), ha demostrado correlacionarse con el estado nutricional global $^{2}$ y varios aspectos de la evolución del paciente, como la morbilidad y mortalidad. En los últimos años se han publicado diversos estudios en los que se muestra una relación paradójica, no esperada (la obesidad es un factor de riesgo clásico y se asocia a un mayor riesgo de muerte y morbilidad cardiovascular en la población general), en el sentido de que un mayor IMC, en niveles de sobrepeso (IMC $>25$ ) y de obesidad franca (IMC $>30$ ) se han relacionado con mejor supervivencia en los pacientes en hemodiálisis ${ }^{3-5}$. Esta relación no ha podido demostrarse tan claramente en los pacientes en diálisis peritoneal6, pero sí se ha observado en otros colectivos de pacientes con enfermedades graves, como insuficiencia cardiaca congestiva, ancianos y pacientes con cáncer o SIDA3.

Aunque no se tiene actualmente una explicación para este hecho, parece que la obesidad puede proteger de los efectos sobre la nutrición de los fenómenos de inflamación, directamente relacionados a su vez con los procedimientos de la hemodiálisis y enfermedades concomitantes frecuentes en los pacientes con IRC (insuficiencia cardiaca, infecciones...), el llamado síndrome MIA, que asocia la malnutrición a la arteriosclerosis, a la inflamación y a la malnutrición?

En los pacientes con IRC avanzada se han hecho menos estudios, a pesar de la importancia que puede tener si se toman las medidas oportunas precozmente para mejorar el pronóstico de los pacientes a lo largo de su estancia en diálisis.

En este trabajo nos hemos propuesto estudiar la influencia del IMC en la supervivencia a los 5 años en pacientes en Prediálisis, tanto en el total del grupo como en el subgrupo de pacientes que inician diálisis.

\section{Material y Métodos}

La muestra estaba formada por todos los pacientes que fueron vistos por primera vez en la consulta de Prediálisis desde en el periodo comprendido entre el 1 de septiembre de 1998 al 31 de octubre de 1999, un total de 160 pacientes. Los criterios de derivación a esta consulta en ese tiempo incluían $\mathrm{Cr}$ sérica superior o igual a $4 \mathrm{mg} / \mathrm{dl} \mathrm{y} / \mathrm{o}$ aclaramiento de creatinina igual o inferior a $20 \mathrm{cc} / \mathrm{m}$. Actualmente este grado de IRC está incluido en los estadios IV (Ccr inferior a $30 \mathrm{cc} / \mathrm{m})$ y V (Ccr inferior a $15 \mathrm{cc} / \mathrm{m})$ siguiendo la clasificación de las Guías DOQI de $2003^{7}$.

Se recogieron los datos de la Historia de Enfermería que recoge las MAM en cada consulta: peso, talla, perímetro del brazo (PB) y pliegue cutáneo del trí- 
ceps (PCT), si bien solo se tuvieron en cuenta las de la primera consulta (basal) y las de la última consulta (final: antes de entrar en diálisis 0 antes del fallecimiento). A partir de estas medias se calcularon:

- IMC: peso ( $\mathrm{Kg}) /$ altura $^{2}$ (metros)

- Circunferencia muscular del brazo (CMB -cm-): PB-0,314*PCT

- Área muscular del brazo (AMB-cm²-): [PB-P $x$ PCT]2/4P

De todas se calculó el porcentaje del percentil 50 para la edad, sexo y altura del paciente según las tablas de la población normal de Alastrué ${ }^{\text {. }}$

Los datos de la situación del paciente a 31 de diciembre de 2004 se recogieron del registro de pacientes de la consulta, llevado asimismo por Enfermería y del Registro de pacientes con IRC del Servicio Andaluz de Salud. Además, la situación de todos los pacientes que habían iniciado hemodiálisis se comprobó a través de los centros periféricos de diálisis.

El análisis de los datos se realizó con el paquete estadístico SPSS 12.

\section{Resultados}

De los 160 pacientes, cuyos datos se muestran en la tabla 1, conocíamos la situación a los 5 años de 157 pacientes: 85 (54\%) permanecían vivos: 19 seguían en Prediálisis, 43 seguían en diálisis y 23 con un trasplante funcionante. De los 72 pacientes fallecidos: 36 fallecieron durante el seguimiento en esta consulta y 36 fallecieron durante su estancia en diálisis; del total de 157 pacientes, 102 (65\%) iniciaron diálisis (4 diálisis peritoneal, resto hemodiálisis).

Se analizó la supervivencia en relación al IMC desde distintos puntos de vista. En primer lugar se comparó ( $U$ de Mann-Withney) la supervivencia como variable cuantitativa (en meses) en pacientes obesos (IMC igual o superior a 30 ) y no obesos, según el valor del IMC basal y el IMC final, tanto en el grupo completo como en el subgrupo que inicia diálisis.

\begin{tabular}{|l|r|}
\hline $\mathrm{n}$ & media \pm de \\
\hline 160 edad & $61 \pm 15$ \\
\hline 160 sexo(mujer/hombre) & $70 / 90$ \\
\hline 160 IMC basal & $28,3 \pm 5,3$ \\
\hline 149 IMC final & $27,7 \pm 5,3$ \\
\hline $155 \% *$ peso basal & $111 \pm 21$ \\
\hline $143 \%$ peso final & $09 \pm 211$ \\
\hline $155 \%$ pct basal & $163 \pm 70$ \\
\hline $136 \%$ pct final & $146 \pm 55$ \\
\hline 154 amb basal & $97 \pm 22$ \\
\hline 135 amb final & $98 \pm 21$ \\
\hline 157 vivo/ exitus a 5 años & $85 / 72$ \\
\hline 157 supervivencia 5 años(meses) & $56 \pm 24$ \\
\hline 104 seguimiento Prediálisis (meses) & $20 \pm 16$ \\
\hline
\end{tabular}

Tabla 1. Datos demográficos, medidas antropométricas y supervivencia. Todos los pacientes.

*\%: porcentaje del percentil 50 de la media estándar según edad, talla y sexo según las Tablas de Alastrué (1).

Basal: datos obtenidos en la primera consulta Prediálisis.

Final: datos obtenidos antes de exitus, antes de la primera diálisis o en la última revisión en consulta para los paciente que siguen en Prediálisis.

En el grupo completo de los 157 pacientes los obesos según la valoración basal (la de la primera consulta) tenían una supervivencia (SV) inferior ( $51 \pm 25$ vs $58 \pm 23$ meses) pero la diferencia no alcanzó la significación estadística $(p=0,09)$; al comparar según el IMC final (peso de la de la última consulta, de la última consulta antes de iniciar diálisis o la última disponible antes del fallecimiento) los pacientes obesos también tenían una SV inferior ( $53 \pm 24$ vs $61 \pm 19$ meses) con significación estadística $(p=0,04)$.

Cuando estudiamos el subgrupo de pacientes que iniciaron diálisis no se alcanzó significación estadística, si bien la SV fue inferior en obesos tanto según el IMC basal $(58 \pm 21$ vs $64 \pm 18$ meses $p=0,1)$ como el IMC final ( $58 \pm 22$ vs $64 \pm 17$ meses $p=0,1)$. 
Asimismo se comparó el IMC, las otras MAM y la edad en el grupo de pacientes que habían fallecido $(n=72)$ resultando solo la edad y el IMC basal con diferencias estadísticamente significativas, siendo los pacientes fallecidos más obesos (IMC $29,3 \pm 5$ vs $27,5 \pm 5 p=0,02)$ y con mayor edad $(70 \pm 10$ vs $54 \pm 15$ años $p=0,001$ ).

Por ultimo se analizó la supervivencia como variable cualitativa en relación a la obesidad con el método de Kaplan-Meier y el test de log-rank aplicándolo al grupo completo así como al subgrupo de los pacientes que inician diálisis. No encontramos diferencias estadísticamente significativas en el total del grupo al tener en cuenta el IMC basal (figura 1) pero si al comparar según el IMC final (figura 2), teniendo los pacientes obesos menor supervivencia $(p=0,03)$; al analizar solo los pacientes que inician diálisis no hay diferencias significativas según el IMC basal ni el final.

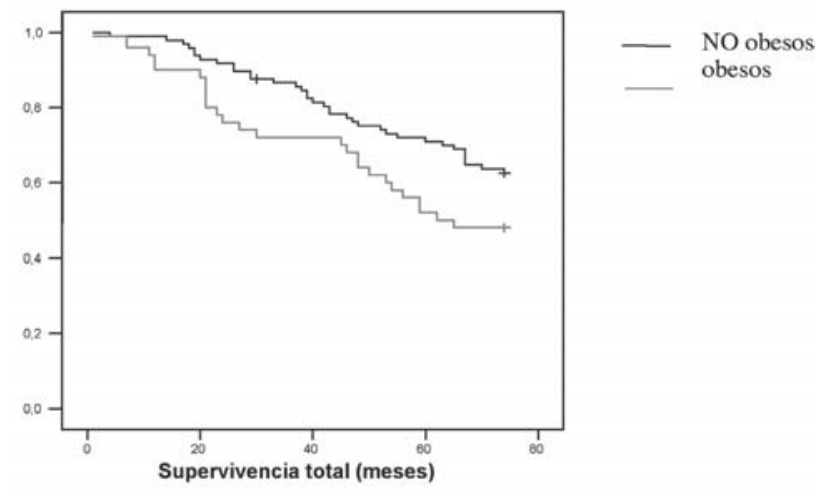

Figura 1. Supervivencia según IMC basal. Todos los pacientes Breslow $p=0,08 ; \log$ rank $p=0,08$.

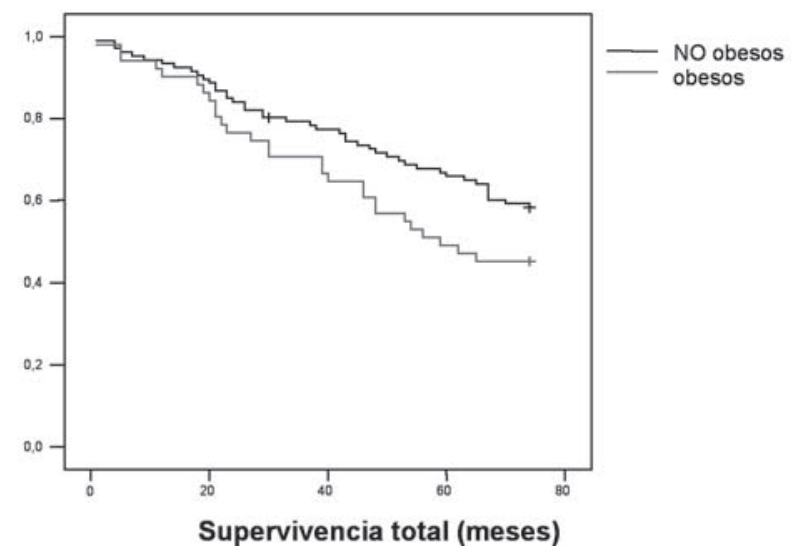

Figura 2. Supervivencia según IMC final. Todos los pacientes Breslow $p=0,05 ; \log$ rank $p=0,03$.

\section{Discusión}

En nuestro estudio hemos encontrado que la obesidad, definida como IMC $\geq 30$, influye negativamente en la supervivencia a los 5 años en nuestra población de pacientes con IRC avanzada tomados en su conjunto y en el subgrupo de pacientes que inician diálisis, mostrando menor supervivencia los pacientes obesos.

Estos resultados concuerdan en parte con un estudio similar realizado en nuestro país ${ }^{8}$ en el que se estudió la influencia de la obesidad en la supervivencia de un grupo de 376 pacientes incidentes en la consulta de Prediálisis durante 5 años. Se observó que aunque tomados en conjunto la supervivencia no varió según el paciente estuviera o no obeso, en el subgrupo de pacientes sin comorbilidad, el IMC superior se asoció a peor supervivencia. Este efecto del IMC, sin embargo, fue neutro para los pacientes con elevados índices de comorbilidad. Es decir, parece que en los individuos con patología, por lo general vascular, severa, el efecto de la obesidad es inferior a la influencia de las otras enfermedades.

Tanto nuestro estudio como el mencionado anteriormente están en contradicción con recientes publicaciones en las que se ha encontrado un efecto positivo de cierto grado de sobrepeso (IMC 25-30) u obesidad franca $(I M C>30)$ en pacientes incidentes o prevalentes con IRC en diálisis ${ }^{3,4,9}$ y en otros subgrupos de pacientes con patología severa 0 ancianos ${ }^{3}$. Esta "paradoja de la obesidad" puede ser explicada en parte al tratarse de estudios trasversales que incluyen pacientes incidentes y prevalentes en diálisis y, como es de esperar, los obesos más enfermos fallecen antes y solo entran en estos estudios los obesos supervivientes. Otra justificación puede ser que se excluyan de estos estudios los pacientes fallecidos en los primeros meses.

La justificación de la discrepancia con nuestros resultados debe estar en primer lugar en la muestra, formada por pacientes con IRC en Prediálisis, en los que el efecto de la obesidad debe ser similar a la población general, es decir, negativo. Sin embargo, si nos ceñimos al subgrupo de pacientes que inician diálisis, que puede considerarse una población incidente en diálisis, la población es comparable y sin embargo la influencia en la supervivencia sigue siendo negativa. 
Otro dato a señalar es el EN de nuestros pacientes, con valores dentro o superiores a la media de la población sana tanto en el compartimiento proteico como calórico, especialmente este último. Es posible que al hallarnos con una población bien nutrida el posible efecto negativo de la desnutrición sobre la evolución en los pacientes con procesos crónicos sería neutralizado y justificaría que los de mayor sobrepeso tuvieran un riesgo de muerte superior, similar a lo que ocurre en la población general.

En nuestro estudio no tenemos sesgo de selección, que se le achaca a otros trabajos, ya que hemos recogido los datos de todos los pacientes que han Ilegado consecutivamente a nuestra consulta de Prediálisis durante un periodo de tiempo determinado, sin exclusiones de pacientes que fallecen precozmente y hemos recogido la evolución a lo largo de los siguientes 5 años de la practica totalidad de los pacientes, que da más valor a nuestros resultados. Asimismo, aunque el subgrupo de nuestros pacientes que inician diálisis es una población homogénea, ya que todos procedían de la consulta Prediálisis, estos resultados no pueden hacerse extensivo a la población global de los pacientes que inician diálisis, en la que un $30-40 \%$ procede de Urgencias, con IRC en fase V no conocida, y que no han sido tratados para la corrección de la anemia, hiperparatiroidismo, acidosis metabólica, etc. previamente, por lo que llegan en peor situación clínica. Es posible que en estos pacientes de diagnostico tardío de la IRC, la obesidad sí marque a los pacientes con mejor estado general inicial, más actividad física, más apetito, más peso, mejor evolución global y finalmente mayor supervivencia.

Podemos concluir que, en nuestro grupo de pacientes, la obesidad, definida como IMC $\geq 30$, influye negativamente en la supervivencia a los 5 años en nuestra población de pacientes con IRC avanzada atendidos en la consulta Prediálisis tomados en su conjunto.

\section{Bibliografía}

1. Alastrué A, Jaurrieta E, Puig P, Abad JM et al. Valoración antropométrica del estado de nutrición: normas y criterios de desnutrición y obesidad. Med Clin 1983; 80: 691-699.

2. Manzano Angua JM, Nieto Granados $M^{a} D$, Sánchez Cornejo $\mathrm{M}^{\mathrm{a}} \mathrm{C}$. Parámetros antropométricos más idóneos para valorar el estado nutricional de los pacientes con insuficiencia renal crónica tratados con hemodiálisis en los centros periféricos. Rev Soc Esp Enferm Nefrol 2.003; 6(3):615.

3. Kalantar-Zadeh K, Abbott KC, Salahudeen AK et al. Survival adventages of obesity in diálisis patients. Am J Clin Nutr 2005; 81: 543-554.

4. Leavy SF, Strawderman RL, Jones CA et al. Simple nutritional indicators as independent predictors of mortality in hemodialysis patients. Am J Kidney Dis 1998; 31: 997-1006.

5. Kopple JD. The phenomenon of altered risk factor patterns or reverse epidemiology in persons with advanced chronic kidney failure. Am J Clin Nutr 2005; 81:1257-66.

6. Abbott KC, Glanton CW, Trespalacios FC et al. Body mass index, dialysis modality and survival; analysis of the United Status Renal Data System Dialysis Morbidity and Mortality Wave II Study. Kidney Int 2004; 65: 597-605.

7. K/DOQI Clinical Practice Guidelines for Chronic Kidney Diseases: evaluation, classification and stratification. Am J Kidney Dis 2002; 39 (supp1): S1-S266.

8. Caravaca F, Martín MV, Barroso S, Arrobas M, Ruiz-Calero R, García MC, Luna E, Sánchez Casado $E$. Obesidad y mortalidad en pacientes con insuficiencia renal avanzada. Nefrología 2004; XXIV, 453-462.

9. Johansen $K L$, Kutner NG, Young B, Chertow GM. Association of body size with health status in patients beginning dialysis. Am J Clin Nutr 2006; 83:543-49. 